\title{
Associations of circulating irisin with 24-h blood pressure, total and visceral fat, and metabolic parameters in young adult hypertensives
}

Tomasz Miazgowski ${ }^{1}$

https://orcid.org/0000-0002-4819-9376

Bartosz Miazgowski²

https://orcid. org/0000-0002-9114-8304

Anna Kaczmarkiewicz ${ }^{2}$

https://orcid. org/0000-0001-8576-6352

Jacek Kopeć ${ }^{3}$

https://orcid.org/0000-0002-2974-7904

\begin{abstract}
Objective: Some experimental and clinical studies suggest a possible role of irisin in central and peripheral regulation of blood pressure. The purpose of the study was to assess the associations between serum irisin levels, total and visceral fat, metabolic parameters, and blood pressure pattern during 24-h monitoring (ABPM). Materials and methods: In 206 patients with essential hypertension receiving standard antihypertensive treatments, we assessed anthropometric indices; serum irisin, blood lipids (total cholesterol, LDL-C, HDL-C, and triglycerides), glucose and insulin; body composition including lean mass and total, visceral, android and gynoid fat using a dual-energy $\mathrm{x}$-ray absorptiometry; ABPM; and Homeostasis Model Assessment-Insulin Resistance (HOMA-IR). Results: Baseline irisin levels were within normal reference ranges and comparable between the genders. There were no significant correlations of irisin with age, anthropometric variables, lipids, HOMA-IR, body composition, as well as 24 -h blood pressure and dipping status. In univariate analysis, age, fat mass and distribution, lipids and glucose, HOMA-IR, and nocturnal blood pressure fall were poor predictors of irisin levels. These neutral associations were not affected by age, gender, and treatment modality. Conclusions: In young adult hypertensives, serum concentration of irisin was within a normal range and not associated with total and regional fat, blood lipids, insulin resistance, as well as 24-h blood pressure and the magnitude of its nocturnal fall. Arch Endocrinol Metab. 2021;65(2):137-43
\end{abstract}

Keywords

Irisin; visceral fat; 24-h blood pressure
${ }^{1}$ Department of Propedeutics of Internal Diseases \& Hypertension, Pomeranian Medical University in Szczecin, Poland

2 Doctoral Study, Pomeranian

Medical University in

Szczecin, Poland

${ }^{3}$ Division of Epidemiology, Biostatistics and Public Health Practice, University of British Columbia, Vancouver, Canada

Correspondence to: Tomasz Miazgowski Department of Propedeutics of Internal Diseases \& Hypertension, Pomeranian Medical University, Unii Lubelskiej 1, 71252 Szczecin, Poland miazgowski@interia.pl

Received on Aug/7/2020 Accepted on Dec/17/2020

DOI: 10.20945/2359-3997000000333

\section{INTRODUCTION}

$\mathrm{I}$ risin - a novel, exercise-induced, myokine produced by proteolytic cleavage of fibronectin type III domain-containing 5 (FNDC5) - is mainly involved in the regulation of energy metabolism by activating thermogenesis to increase energy expenditure (1). In addition, it has been suggested that the myokine might be associated with glucose and lipid metabolism, insulin sensitivity (2), oxidative stress (3), and variations in body weight (4). Some reports demonstrated an association of irisin with systolic (SBP) (5-7) and diastolic (DBP) (5-8) blood pressure, but other yielded conflicting results $(9,10)$. In experimental rat models, administration of exogenous recombinant human irisin to the $3^{\text {rd }}$ brain ventricle activated neurons in the paraventricular nuclei of the hypothalamus resulting in the increases in SBP, DBP and cardiac contractibility. In contrast, when it was given intravenously, blood pressure was lowered but cardiac contractibility was not affected (11). These findings suggest that irisin can affect blood pressure by two different mechanisms: central, involving adrenergic sympathetic activity and increased vasopressin release, and peripheral - by a direct vasodilation through ATP-sensitive potassium channel and/or amelioration of endothelial dysfunction $(11,12)$. Based on these observations, we theorized that that irisin might modulate diurnal blood pressure profile not only in rodents but also in humans. The purpose of this study was to investigate whether circulating irisin is independently associated with 24-h SBP, DBP, and physiological blood pressure nocturnal fall in young 
adult hypertensives. We also examined associations between irisin, metabolic parameters and fat distribution including visceral adipose tissue, which is known to contribute to many detrimental metabolic effects.

\section{MATERIALS AND METHODS}

\section{Study participants}

We included 206 young adult subjects (132 males; 74 females) aged 18-35 years who were diagnosed in our tertiary care unit with primary hypertension. The subjects with endocrine disorders, history of malignancy, nutrition disorders, and rapid weight changes ( $\geq 5 \mathrm{~kg}$ ) within the last 12 months, as well as pregnant women and patients receiving medications or dietary supplements known to affect body composition (insulin, glucocorticoids, anabolic steroids, protein supplements, etc.) were excluded. Patients with secondary or spurious isolated systolic hypertension were also excluded. All subjects received standard antihypertensive drugs $(13,14)$ including reninangiotensin-aldosterone system (RAAS) inhibitors: angiotensin-converting enzyme or ATl receptor inhibitors (men only; $\mathrm{n}=114$ ); beta-blockers $(\mathrm{n}=42)$; calcium channel blockers (CCBs; $n=100$ ); thiazidelike diuretics $(n=15)$; and alfa ${ }_{1}$-blockers $(n=12)$ given either as a monotherapy or in combinations. The study complied with all applicable institutional regulations regarding the ethical use of human volunteers in research and the terms of the Declaration of Helsinki. The Pomeranian Medical University Ethics Committee approved the study protocol, and all participants gave their written consent.

\section{Procedures}

Height (rounded to the nearest $0.5 \mathrm{~cm}$ ), weight, waist circumference (WC) and hip circumference (HC) were measured. Body mass index (BMI) was calculated as $\mathrm{BMI}=$ weight/height ${ }^{2}$. Waist-to-hip ratio (WHR) was calculated as WHR=WC/HC. A 24-h ambulatory blood pressure monitoring (ABPM) was recorded using the Spacelabs device (model 90207; Spacelabs Healthcare; WA, USA). Automated blood pressure measurements were performed every $20 \mathrm{~min}$ during the day and every 30 min during nighttime (between 22.00 and 06.00). Based on a percent difference between daytime and nocturnal mean SBP, we identified the following blood pressure patterns: dipping (nocturnal SBP fall by $10-20 \%$ ), non- dipping $(<10 \%)$, extreme dipping $(>20 \%)$ and reverse dipping (nocturnal SBP higher than daytime) $(13,14)$.

\section{Biochemical assessment}

After an overnight fast, lipid profiles including serum levels of triglycerides (TG) and total, low-densitylipoprotein (LDL-C)-, and high-density-lipoprotein (HDL-C)-cholesterol, glucose, and insulin were assessed. From insulin and glucose measurements, a Homeostatic-Model-Assessment Insulin Resistance Index (HOMA-IR) was calculated. Serum irisin was assessed by ELISA using recombinant antibodies (Irisin Recombinant Human, Mouse, Rat, Canine; Phoenix Pharmaceuticals Inc., USA; normal range provided by the manufacturer: $5.8-23.2 \mathrm{ng} / \mathrm{mL}$ ).

\section{Body composition}

Body composition was assessed using dual-energy X-ray absorptiometry (DXA) (GE Healthcare Lunar Prodigy Advance; Madison, WI, USA) using the automatic whole-body scan mode. We analyzed total body (TBF), android, and gynoid fat, as well as lean mass (LM), which in DXA is a surrogate measure of muscle mass. Visceral fat (VF) was computed by the CoreScan application dedicated to GE Healthcare DXA devices. Body composition parameters were analyzed using age-, gender-, race-, and instrument-specific reference values for $\operatorname{VF}(15,16)$ and TBF (17).

\section{Definitions}

Traditional metabolic risk factors were defined using the following International Diabetes Federation diagnostic criteria for metabolic syndrome in populations of European descent (18): (a) WC $\geq 94 \mathrm{~cm}$ in men and $\geq 80 \mathrm{~cm}$ in women; (b) fasting glucose $\geq 100$ $\mathrm{mg} / \mathrm{dL}(\geq 5.6 \mathrm{mmol} / \mathrm{L})$; (c) HDL-C $<40 \mathrm{mg} / \mathrm{dL}$ $(<1.3 \mathrm{mmol} / \mathrm{L})$ in men and $<50 \mathrm{mg} / \mathrm{dL}(<1.29$ $\mathrm{mmol} / \mathrm{L}$ ) in women; and (d) TG $\geq 150 \mathrm{mg} / \mathrm{dL}$ (1.7 $\mathrm{mmol} / \mathrm{L})$. For the definition of high total cholesterol and LDL-C, we used the following cut-offs: $>200$ $\mathrm{mg} / \mathrm{dL}(>5.17 \mathrm{mmol} / \mathrm{L})$ and $\geq 115 \mathrm{mg} / \mathrm{dL}(2.97$ $\mathrm{mmol} / \mathrm{L})$, respectively. From (19) we used the value of HOMA-IR of $\geq 2.5$ as a marker for insulin resistance.

\section{Statistical analyses}

Descriptive statistics included means \pm standard deviation (SD) for continuous variables and frequency distributions for categorical variables. Variables with 
normal distribution were compared using parametric Student's $t$ tests; otherwise, non-parametric MannWhitney U-tests and Kruskall-Wallis tests were used. Differences in serum irisin levels between patients receiving monotherapy from the main three antihypertensive drug classes were compared using ANOVA. Correlations between pairs of quantitative variables were assessed using Pearson's linear correlations or Spearman's rho correlations for normally and nonnormally distributed variables, respectively. Using a post-hoc analysis, the statistical power of the study with 206 subjects was sufficient to detect with $80 \%$ probability the true effect size of an association between quantitative variables corresponding to a correlation coefficient of 0.20. Statistical analyses were performed using Statistica (StatSoft, Poland; version 13.0).

\section{RESULTS}

Patient characteristics are shown in Table 1 and Figure 1. Approximately $50 \%$ of patients had high BMI $\left(>25.0 \mathrm{~kg} / \mathrm{m}^{2}\right), \mathrm{WC}, \mathrm{VF}$, and TBF. In approximately

Table 1. Descriptive statistics of the study population

\begin{tabular}{|c|c|c|c|c|}
\hline & All $(n=206)$ & Men $(n=132)$ & Women $(n=74)$ & $\mathbf{P}$ \\
\hline \multicolumn{5}{|l|}{ Anthropometric variables } \\
\hline Age (years) & $27.40 \pm 5.12$ & $26.75 \pm 5.13$ & $27.55 \pm 4.92$ & 0.0509 \\
\hline Weight (kg) & $83.24 \pm 17.9$ & $89.95 \pm 14.6$ & $71.27 \pm 17.1$ & 0.0001 \\
\hline Body mass index $\left(\mathrm{kg} / \mathrm{m}^{2}\right)$ & $27.20 \pm 4.90$ & $27.84 \pm 4.34$ & $26.05 \pm 5.73$ & 0.0022 \\
\hline Waist circumference (cm) & $93.99 \pm 14.4$ & $97.61 \pm 13.1$ & $87.54 \pm 14.4$ & 0.0001 \\
\hline Hip circumference (cm) & $100.0 \pm 11.1$ & $96.62 \pm 13.5$ & $101.9 \pm 9.03$ & 0.0051 \\
\hline Waist-to-hip ratio & $0.938 \pm 0.10$ & $0.955 \pm 0.11$ & $0.906 \pm 0.10$ & 0.0001 \\
\hline Family history of hypertension (n) & $97(47.08 \%)$ & $60(45.45 \%)$ & $37(50.0 \%)$ & 0.6301 \\
\hline \multicolumn{5}{|l|}{ 24-h Blood pressure } \\
\hline Systolic blood pressure (mmHg) & $131.2 \pm 14.7$ & $132.6 \pm 14.7$ & $128.7 \pm 14.5$ & 0.0519 \\
\hline Daytime & $135.8 \pm 14.9$ & $136.8 \pm 14.9$ & $133.9 \pm 14.7$ & 0.0601 \\
\hline Nighttime & $122.7 \pm 13.7$ & $123.4 \pm 13.7$ & $121.7 \pm 13.6$ & 0.0945 \\
\hline Diastolic blood pressure (mmHg) & $77.31 \pm 11.4$ & $75.00 \pm 10.0$ & $81.43 \pm 12.6$ & 0.0014 \\
\hline Daytime & $83.24 \pm 12.1$ & $80.13 \pm 11.9$ & $85.90 \pm 12.9$ & 0.0142 \\
\hline Nighttime & $74.01 \pm 11.3$ & $72.06 \pm 11.2$ & $77.71 \pm 11.4$ & 0.0356 \\
\hline Nocturnal blood pressure fall (\%) & $-10.28 \pm 4.81$ & $-10.80 \pm 4.73$ & $-9.400 \pm 4.92$ & 0.0182 \\
\hline \multicolumn{5}{|l|}{ Biochemical assessment } \\
\hline Insulin ( $\mu \mid \mathrm{U} / \mathrm{mL})$ & $12.59 \pm 12.72$ & $12.96 \pm 15.01$ & $11.89 \pm 6.62$ & 0.5121 \\
\hline Glucose (mg/dL) & $88.96 \pm 9.73$ & $89.85 \pm 9.90$ & $87.32 \pm 9.31$ & 0.1180 \\
\hline HOMA-IR & $2.853 \pm 3.72$ & $2.991 \pm 4.52$ & $2.598 \pm 1.63$ & 0.7751 \\
\hline Total cholesterol (mg/dL) & $181.7 \pm 36.7$ & $186.5 \pm 38.6$ & $172.7 \pm 31.1$ & 0.0283 \\
\hline HDL-cholesterol (mg/dL) & $51.86 \pm 14.5$ & $47.81 \pm 12.8$ & $59.38 \pm 14.7$ & 0.0001 \\
\hline LDL-cholesterol (mg/dL) & $116.4 \pm 33.9$ & $122.2 \pm 33.9$ & $105.7 \pm 31.2$ & 0.0024 \\
\hline Triglycerides (mg/dL) & $136.5 \pm 71.3$ & $147.9 \pm 75.2$ & $115.2 \pm 58.1$ & 0.0090 \\
\hline Irisin (ng/mL) & $10.46 \pm 3.23$ & $10.25 \pm 2.66$ & $10.54 \pm 2.68$ & 0.3110 \\
\hline \multicolumn{5}{|l|}{ Body composition } \\
\hline Total fat (kg) & $26.54 \pm 10.6$ & $26.43 \pm 10.0$ & $26.73 \pm 11.5$ & 0.7750 \\
\hline Total fat (\%) & $32.42 \pm 8.27$ & $29.77 \pm 7.38$ & $37.14 \pm 7.67$ & 0.0001 \\
\hline Android fat (kg) & $2.446 \pm 1.41$ & $2.571 \pm 1.38$ & $2.222 \pm 1.43$ & 0.0412 \\
\hline Gynoid fat (kg) & $4.406 \pm 2.26$ & $4.101 \pm 1.43$ & $4.948 \pm 2.27$ & 0.0449 \\
\hline Visceral fat (kg) & $0.920 \pm 0.70$ & $1.110 \pm 0.71$ & $0.580 \pm 0.54$ & 0.0001 \\
\hline Lean mass (kg) & $53.73 \pm 10.8$ & $59.95 \pm 7.23$ & $42.63 \pm 6.45$ & 0.0001 \\
\hline
\end{tabular}

P-value refers to comparison between women and men. 


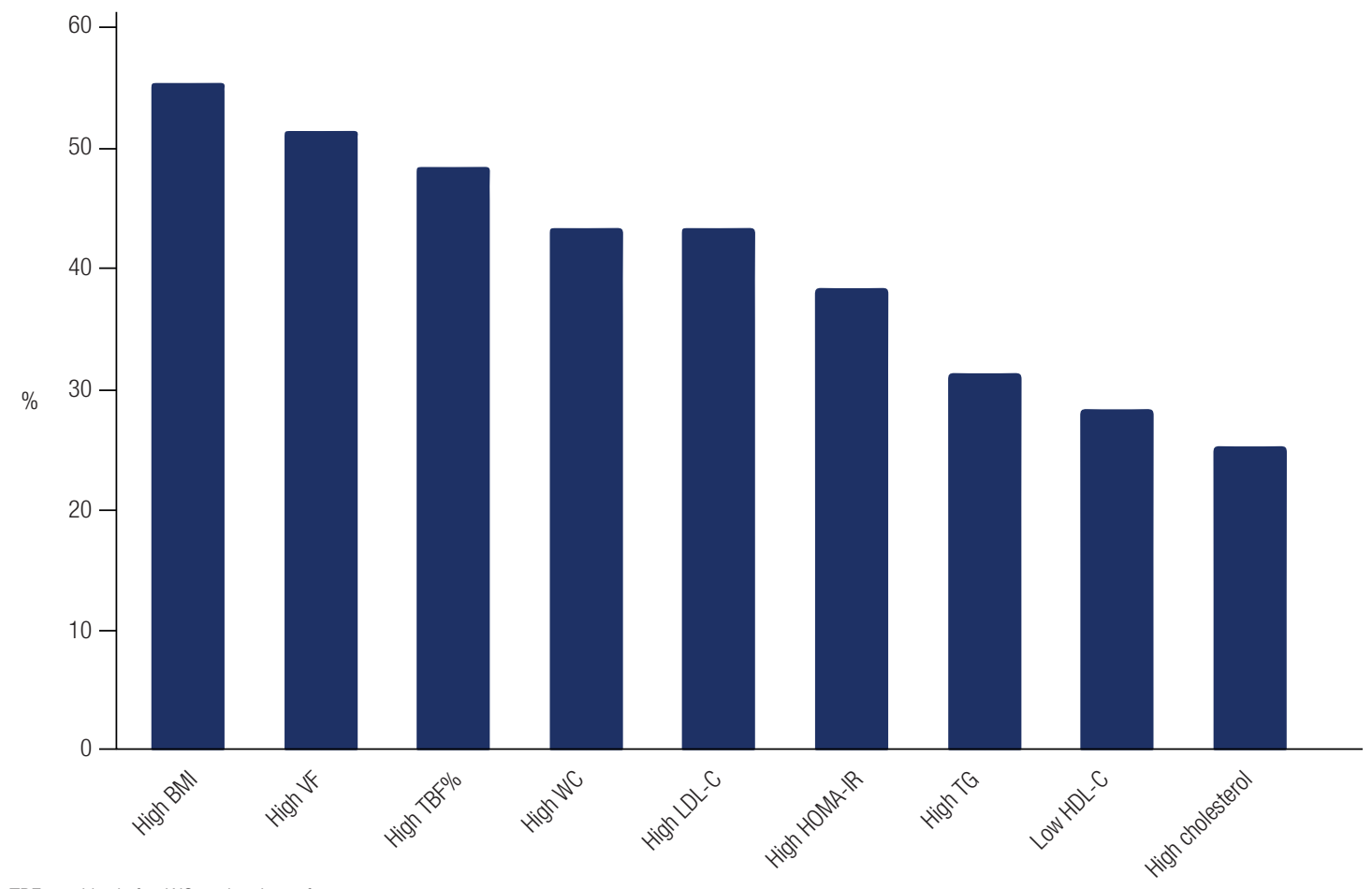

VF: visceral fat; TBF: total body fat; WC: waist circumference.

Figure 1. Frequency distribution of metabolic risk factors in the study population.

$20-25 \%$ of patients, mean values of SBP and DBP measured during a 24 -h period, daytime and nighttime, were above recommended targets $(13,14)$. In addition, almost $50 \%$ of males and $35 \%$ of females displayed a non-dipping pattern of blood pressure.

Serum irisin levels were within normal reference ranges and comparable between males and females. There were no significant differences in irisin levels between the groups receiving medications from three main antihypertensive drug classes in monotherapy $(10.33 \pm 2.62 \mathrm{ng} / \mathrm{mL}, 10.49 \pm 2.71 \mathrm{ng} / \mathrm{mL}$ and $10.22 \pm 2.76 \mathrm{ng} / \mathrm{mL}$ for RAAS inhibitors, CCBs, and beta-blockers, respectively). Similarly, differences in irisin serum concentrations between the patients with excess TBF\% and VF defined as the values $>1$ SD from age- and gender-specific cut offs (for TBF\%: $>37.7 \%$ in females and $>30.6 \%$ in males (17); for VF: $>0.484$ $\mathrm{kg}$ and $>0.993 \mathrm{~kg}$ in females and males, respectively (15)), as well as between dippers and non-dippers were insignificant (Table 2).

In the whole group of patients (males and females combined), no significant correlations were found between irisin and ABPM (Figure 2) and body composition (Figure 3), as well as anthropometric measurements, blood lipids, insulin, glucose and
HOMA-IR. Similarly, irisin was not associated with age, BMI, fat distribution, and other study variables in univariate regression (Table 3 ).

\section{DISCUSSION}

The association of circulating irisin levels with blood pressure in humans has not yet been fully elucidated. Using different populations, some studies found irisin to be positively correlated with both SBP and DBP (57 ) or solely $\mathrm{DBP}(8)$, while other found no association $(9,10)$. However, in all these reports, blood pressure was measured manually using automated devices, what in comparison of clinical studies can be a source of potential biases caused by random and systematic measurement errors (14,20-22). Instead, we measured blood pressure using ABPM method, which, in comparison to manual methods, is less influenced by inter-observer and inter-device variability. In addition, ABPM allows to perform a 24-hour measurement of blood pressure and its circadian patterns. Using ABPM, we found irisin not to be associated with SBP and DBP or the magnitude of SDP nocturnal fall. Moreover, these neutral associations were not affected by age, gender, and treatment modality. As this is the first study 
Table 2. Serum irisin in relations to TBF, VF, BMl and dipping status

\begin{tabular}{|c|c|c|}
\hline & Irisin (ng/mL) & $\mathbf{P}$ \\
\hline TBF\% within normal ranges $(n=108)$ & $10.09 \pm 2.54$ & 0.3049 \\
\hline TBF\% $>37.7 \%$ (women); $>30.6 \%$ (men) $(\mathrm{n}=98)$ & $10.46 \pm 2.62$ & \\
\hline VF within normal ranges $(n=103)$ & $10.11 \pm 2.49$ & 0.2786 \\
\hline VF $>0.484 \mathrm{~kg}$ (women); $>0.993 \mathrm{~kg}$ (men) $(\mathrm{n}=103)$ & $10.50 \pm 2.66$ & \\
\hline Dippers $(n=123)$ & $10.61 \pm 2.64$ & 0.2943 \\
\hline Non-dippers ( $n=83$ ) & $10.23 \pm 2.55$ & \\
\hline BMl within normal ranges $(n=93)$ & $9.831 \pm 2.56$ & 0.0737 \\
\hline $\mathrm{BMl} \geq 25.0 \mathrm{~kg} / \mathrm{m}^{2}(\mathrm{n}=113)$ & $10.48 \pm 2.60$ & \\
\hline
\end{tabular}

TBF: total body fat; VF: visceral fat; BMI: body mass index.

Table 3. Univariate regression analysis

\begin{tabular}{|c|c|c|c|}
\hline Irisin (ng/mL) & $b$ & SE & $\mathbf{P}$ \\
\hline Age (years) & -0.0657 & 0.0626 & 0.2981 \\
\hline Body mass index $\left(\mathrm{kg} / \mathrm{m}^{2}\right)$ & -0.1899 & 0.4112 & 0.6456 \\
\hline Waist circumference $(\mathrm{cm})$ & 0.0510 & 0.1246 & 0.8319 \\
\hline Hip circumference (cm) & 0.0800 & 0.2390 & 0.7398 \\
\hline 24-h Systolic blood pressure (mmHg) & 0.1256 & 0.3236 & 0.5621 \\
\hline 24-h Diastolic blood pressure (mmHg) & 0.1425 & 0.2986 & 0.4132 \\
\hline Nocturnal blood pressure fall (\%) & 0.1235 & 0.0322 & 0.2365 \\
\hline Insulin (mlU/mL) & 0.1329 & 0.2566 & 0.0928 \\
\hline Glucose (mg/dL) & -0.1098 & 0.0999 & 0.1212 \\
\hline HOMA-IR & 0.0009 & 0.7901 & 0.9900 \\
\hline HDL-cholesterol (mg/dL) & 0.0388 & 0.0097 & 0.6935 \\
\hline LDL-cholesterol (mg/dL) & 0.0325 & 0.2490 & 0.1967 \\
\hline Triglycerides (mg/dL) & 0.0120 & 0.1280 & 0.3517 \\
\hline Total fat (\%) & -0.1230 & 0.3180 & 0.6992 \\
\hline Android fat (kg) & 0.0942 & 0.0689 & 0.1425 \\
\hline Gynoid fat (kg) & 0.0067 & 0.0086 & 0.7224 \\
\hline Visceral fat (kg) & 0.0203 & 0.0243 & 0.4074 \\
\hline Lean mass $(\mathrm{kg})$ & 0.0007 & 0.0005 & 0.8978 \\
\hline
\end{tabular}

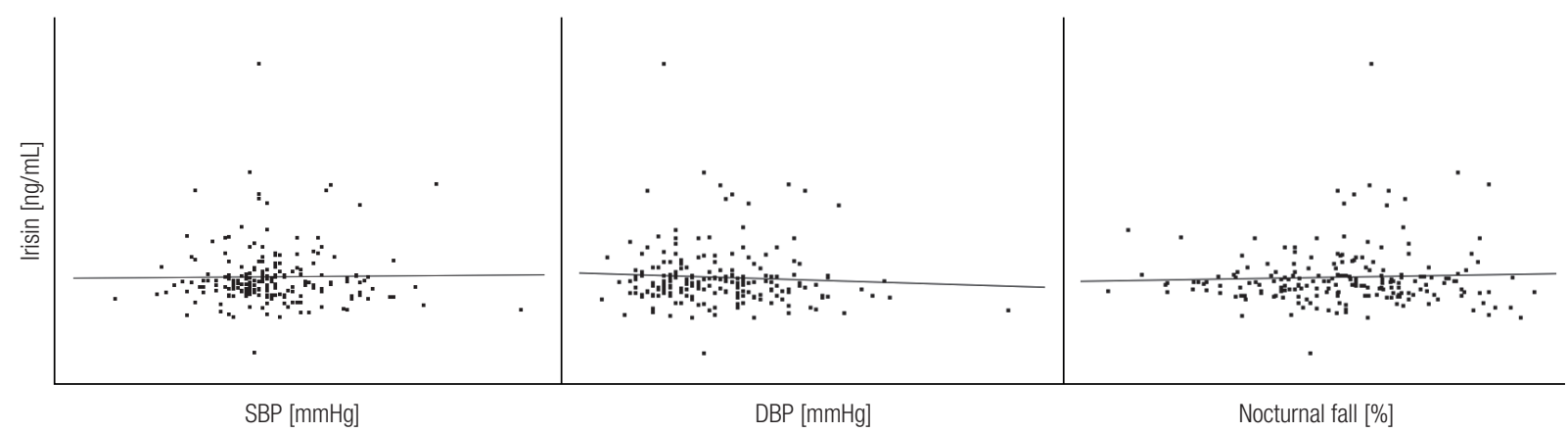

Figure 2. Correlations of irisin with systolic and diastolic blood pressure and nocturnal SBP fall. 


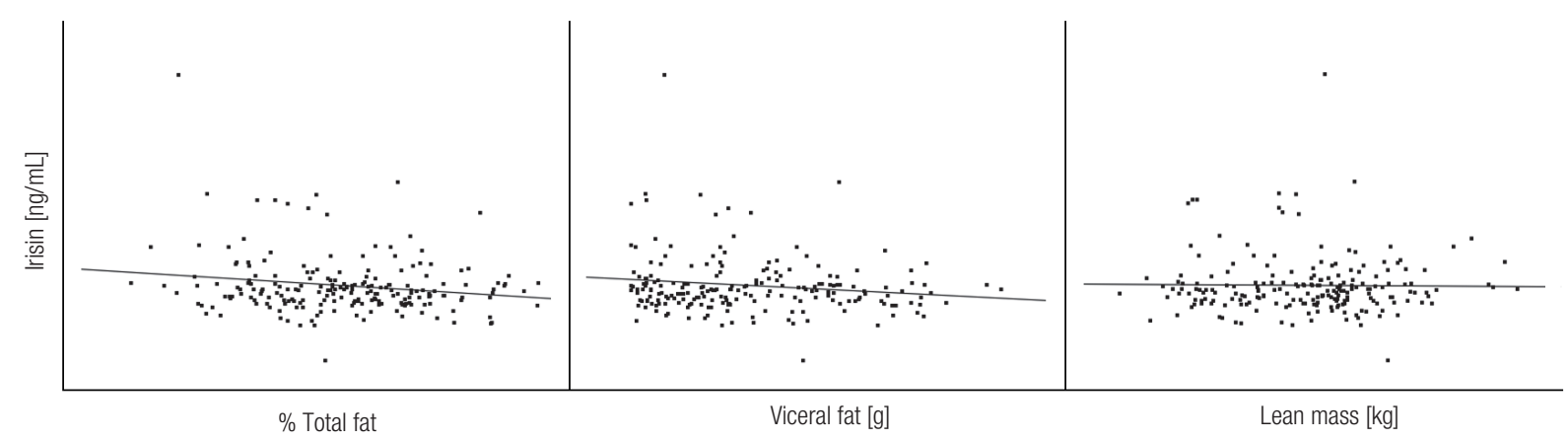

Figure 3. Correlations of irisin with total fat, visceral fat and lean mass.

that assessed irisin associations with blood pressure measured by ABPM, we can only speculate that the myokine, which is released in response to intensive exercising, may exert only a very short-term rather than longer systemic effects on blood pressure, and hence is not detectable in ABPM. This speculation might be supported by experimental data, in which acute central and peripheral administration of irisin that mimics exercise-induced irisin rise can temporary influence arterial blood pressure (11). In addition, intravenous injection of irisin in spontaneous hypertensive rats effectively reduced blood pressure likely via activation of nuclear factor E2-related factor-2 (23), suggesting its potential use in human hypertension.

We could not demonstrate the associations of irisin with body fat and its compartments, as well as obesity-dedicated measures such as BMI, waist and hip circumferences and waist-to-hip ratio. In addition, a novel finding of this study was that VF, which is known to be involved in the development of atherogenic lipid profiles, abnormal glucose tolerance, elevated blood pressure, and other abnormalities frequently related to metabolic syndrome $(16,24)$ was not associated with irisin. Similarly, the myokine levels were not associated with glucose, insulin, HOMA-IR, and blood lipids. In earlier reports, both positive and negative correlations, as well as the lack of correlation between irisin concentrations and BMI, fat mass, lean mass, and other anthropometric parameters were found (6,25-27). Likewise, contradictory evidence was found for the association of irisin plasma levels with cardiovascular and metabolic parameters such as glucose, insulin, HOMA-IR, HDL-C, LDL-C, and TG levels in healthy individuals, as well as in those with obesity and the metabolic syndrome $(4-6,9,10,26,27)$; however, it remains unclear whether these associations reflect a true cause-effect relationship.

Our study had some limitations. Firstly, the study is lacking a control group. This is because we investigated the association of irisin with nocturnal fall in blood pressure, and as non-dipping blood pressure is rare among healthy individuals, we focused on a group of young hypertensives, which comprised both dippers and non-dippers. Secondly, we did not assess the level of physical activity. However, although some studies proposed irisin to be an exercise hormone (28), other questioned the exercise-induced biological relevance of this myokine $(25,29)$ suggesting that other, unknown factors may be involved in the regulation of exerciseinduced irisin effects (30). Finally, we assessed irisin levels using a commercial ELISA kit but the accuracy of this method has been questioned. As human FNDC5, which is the precursor of irisin, is mainly translated from its non-canonical start codon, it has been claimed that human irisin antibodies commonly used in ELISA lack required specificity $(31,32)$.

In summary, our study demonstrated that in young adults with hypertension serum concentration of irisin was normal and not associated with total and regional fat, blood lipids, insulin resistance, as well as 24 -h blood pressure and its nocturnal fall.

Acknowledgements: this work was supported by the Pomeranian Medical University (Grant for Young Researchers No. MB-315201/16) and Polish Osteoporosis Foundation BO in Szczecin. The funding organizations had no role in the design, analysis and decision to submit the manuscript for publication.

Disclosure: no potential conflict of interest relevant to this article was reported. 


\section{REFERENCES}

1. Polyzos SA, Kountouras J, Shields K, Mantzoros CS. Irisin: a renaissance in metabolism? Metabolism. 2013;62(8):1037-44.

2. $\mathrm{Ye} X$, Shen $Y, N i \mathrm{C}, \mathrm{Ye} J$, Xin Y, Zhang W, et al. Irisin reverses insulin resistance in $\mathrm{C} 2 \mathrm{C} 12$ cells via the p38-MAPK-PGC-1a pathway. Peptides. 2019;119:170120.

3. Hassan II, Hassan AB, Rajab HA, Saadi FS, Abdulah DM, Abdul Majeed $A A$, et al. Association of irisin and oxidative stress with biochemical parameters in patients with metabolic syndrome. Horm Mol Biol Clin Investig. 2019;39(1):20190009.

4. Jia J, Yu F, Wei WP, Yang P, Zhang R, Sheng Y, et al. Relationship between circulating irisin levels and overweight/obesity: A metaanalysis. World J Clin Cases. 2019;7(12):1444-55.

5. De Meneck F, Victorino de Souza L, Oliveira V, do Franco MC. High irisin levels in overweight/obese children and its positive correlation with metabolic profile, blood pressure, and endothelial progenitor cells. Nutr Metab Cardiovasc Dis. 2018;28(7):756-64.

6. Park KH, Zaichenko L, Brinkoetter M, Thakkar B, Sahin-Efe A, Joung $\mathrm{KE}$, et al. Circulating irisin in relation to insulin resistance and the metabolic syndrome. J Clin Endocrinol Metab. 2013;98(12):4899-907.

7. Chang CL, Huang SY, Hsu Y, Chin TH, Soong Y. The serum level of irisin, but not asprosin, is abnormal in polycystic ovary syndrome patients. Sci Rep. 2019;9:4469.

8. Liu JJ, Wong MD, Toy WC, Tan CS, Liu S, Ng XW, et al. Lower circulating irisin is associated with type 2 diabetes mellitus. $\mathrm{J}$ Diabetes Complications. 2013;27(4):365-9.

9. Yan B, Shi X, Zhang H, Pan L, Ma Z, Liu S, et al. Association of serum irisin with metabolic syndrome in obese Chinese adults. PLoS One. 2014;9(4):e94235.

10. Ebert T, Focke D, Petroff D, Wurst U, Richter J, Bachmann A, et al. Serum levels of the myokine irisin in relation to metabolic and renal function. Eur J Endocrinol. 2014;170(4):501-6.

11. Zhang W, Chang L, Zhang C, Zhang R, Li Z, Chai B, et al. Central and peripheral irisin differentially regulate blood pressure. Cardiovasc Drugs Ther. 2015;29(2):121-7.

12. Fu J, Han Y, Wang J, Liu Y, Zheng S, Zhou L, et al. Irisin lowers blood pressure by improvement of endothelial dysfunction via AMPK-Akt-eNOS-NO pathway in the spontaneously hypertensive rat. J Am Heart Assoc. 2016;5(11):e003433.

13. Williams B, Mancia G, Spiering W, Agabiti Rosei E, Azizi M, Burnier M, et al.; ESC Scientific Document Group. 2018 ESC/ESH Guidelines for the management of arterial hypertension. Eur Heart J. 2018;39(33):3021-104.

14. Chobanian AV, Bakris GL, Black HR, Cushman WC, Green LA, Izzo $\mathrm{JL} \mathrm{Jr}$, et al. The seventh report of the Joint National Committee on Prevention, Detection, Evaluation, and Treatment of High Blood Pressure: the JNC 7 report. JAMA. 2003;289(19):2460-72.

15. Miazgowski T, Kucharski R, Sołtysiak M, Taszarek O, Miazgowski B, Widecka K. Visceral fat reference values derived from healthy European men and women aged 20-30 years using GE Healthcare dual-energy x-ray absorptiometry. PLoS One. 2017;12(7):e0180614.

16. Miazgowski T, Krzyżanowska-Świniarska B, Dziwura-Ogonowska $\mathrm{J}$, Widecka $\mathrm{K}$. The associations between cardiometabolic risk factors and visceral fat measured by a new dual-energy X-ray absorptiometry-derived method in lean healthy Caucasian women. Endocrine 2014;47:500-5.

17. Imboden MT, Welch WA, Swartz AM, Montoye AH, Finch HW, Harber MP, et al. Reference standards for body fat measures using GE dual energy x-ray absorptiometry in Caucasian adults. PLoS One. 2017;12(4):e0175110.
18. Alberti KG, Eckel RH, Grundy SM, Zimmet PZ, Cleeman Jl, Donato KA, et al.; International Diabetes Federation Task Force on Epidemiology and Prevention; Hational Heart, Lung, and Blood Institute; American Heart Association; World Heart Federation; International Atherosclerosis Society; International Association for the Study of Obesity. Harmonizing the metabolic syndrome: a joint interim statement of the International Diabetes Federation Task Force on Epidemiology and Prevention; National Heart, Lung, and Blood Institute; American Heart Association; World Heart Federation; International Atherosclerosis Society; and International Association for the Study of Obesity. Circulation. 2009;120(16):1640-5.

19. Tang $\mathrm{Q}$, Li X, Song $\mathrm{P}, \mathrm{Xu}$ L. Optimal cut-off values for the homeostasis model assessment of insulin resistance (HOMAIR) and pre-diabetes screening: Developments in research and prospects for the future. Drug Discov Ther. 2015;9(6):380-5.

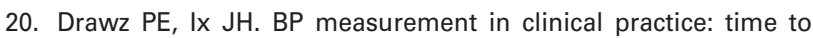
SPRINT to guideline-recommended protocols. J Am Soc Nephrol. 2018;29(2):383-8.

21. Kallioinen N, Hill A, Horswill MS, Ward HE, Watson MO. Sources of inaccuracy in the measurement of adult patients' resting blood pressure in clinical settings. J Hypertens. 2017;35(3):421-41.

22. Hutcheon JA, Chiolero A, Hanley JA. Random measurement error and regression dilution bias. BMJ. 2010;340:c2289.

23. Huo CJ, Yu XJ, Sun YJ, Li HB, Su Q, Bai J, et al. Irisin lowers blood pressure by activating the Nrf2 signaling pathway in the hypothalamic paraventricular nucleus of spontaneously hypertensive rats. Toxicol Appl Pharmacol. 2020;394:114953.

24. Kurniawan LB, Bahrun U, Hatta M, Arif M. Body mass, total body fat percentage, and visceral fat level predict insulin resistance better than waist circumference and body mass index in healthy young male adults in Indonesia. J Clin Med. 2018;7(5):E96.

25. Ebert T, Kralisch S, Wurst U, Scholz M, Stumvoll M, Kovacs $P$, et al. Association of metabolic parameters and rs726344 in FNDC5 with serum irisin concentrations. Int J Obes (Lond). 2016;40(2):260-5.

26. Elizondo-Montemayor L, Mendoza-Lara G, Gutierrez-DelBosque G, Peschard-Franco M, Nieblas B, Garcia-Rivas G. Relationship of circulating irisin with body composition, physical activity, and cardiovascular and metabolic disorders in the pediatric population. Int J Mol Sci. 2018;19(12):3727.

27. Sanchis-Gomar F, Alis R, Pareja-Galeano H, Sola E, Victor VM, Rocha $\mathrm{M}$, et al. Circulating irisin levels are not correlated with $\mathrm{BMI}$, age, and other biological parameters in obese and diabetic patients. Endocrine. 2014;46(3):674-7.

28. Dundar A, Kocahan S, Sahin L. Associations of apelin, leptin, irisin, ghrelin, insulin, glucose levels, and lipid parameters with physical activity during eight weeks of regular exercise training. Arch Physiol Biochem. 2019 Jul 10:1-5.

29. He Z, Tian Y, Valenzuela PL, Huang C, Zhao J, Hong $P$, et al. Myokine/adipokine response to "aerobic" exercise: is it just a matter of exercise load? Front Physiol. 2019;10:691.

30. Czarkowska-Paczek B, Zendzian-Piotrowska M, Gala K, Sobol M, Paczek $L$. One session of exercise or endurance training does not influence serum levels of irisin in rats. J Physiol Pharmacol. 2014;65(3):449-54.

31. Raschke S, Elsen M, Gassenhuber H, Sommerfeld M, Schwahn U, Brockmann B, et al. Evidence against a beneficial effect of irisin in humans. PLoS One. 2013;8(9):e73680.

32. Albrecht $E$, Schering $L$, Buck F, Vlach $K$, Schober HC, Drevon CA, et al. Irisin: Still chasing shadows. Mol Metab. 2020;34:124-35. 